\title{
Physical distribution of SINE elements in the chromosomes of Atlantic salmon and rainbow trout
}

\author{
JULIANA PEREZ, EVA GARCIA-VAZQUEZ* \& PALOMA MORAN \\ Departamento de Biología Funcional (Area de Genética), Facultad de Medicina, C/Julián Clavería s/n, Universidad \\ de Oviedo, Oviedo 33006, Spain
}

\begin{abstract}
SINE sequences are interspersed throughout virtually all eukaryotic genomes. In the family Salmonidae three families of SINEs have been identified. In this work we have attempted to characterize and locate by means of fluorescent in situ hybridization (FISH) one of these families (HpaI) in the genome of Atlantic salmon (Salmo salar) and rainbow trout (Oncorhynchus mykiss). Our results show that these SINEs are interspersed throughout all the chromosome pairs except for the heterochromatin-positive areas, generating a banding pattern that could be useful for chromosome identification in both species.
\end{abstract}

Keywords: chromosome location, fluorescent in situ hybridization, HpaI, Oncorhynchus mykiss, Salmo salar, SINE.

\section{Introduction}

Highly repetitive sequences are interspersed throughout virtually all eukaryotic genomes. They can be classified into two categories based on their size: long interspersed repetitive elements (LINEs) and short interspersed repetitive elements (SINEs). They have been isolated from the genomes of many multicellular organisms from invertebrates to vertebrates (Ohshima et al., 1993; Okada \& Ohshima, 1995). SINEs are typically less than 500 base pairs in length and can be unique to a taxonomic rank, for example a family, a genus or a few species (Smit, 1996). Almost all SINEs reported to date are derived from tRNA (Okada, 1991a,b; Okada \& Ohshima, 1995), with the exception of the primate $A l u$ and the rodent B1 families which are derived from 7SL RNA (Ullu \& Tschudi, 1984). SINEs are believed to be amplified by a process of retrotransposition (Singer, 1982). In contrast to DNA transposable elements, SINEs appear to be inserted irreversibly and thus they should serve as ideal evolutionary and phylogenetic markers (Okada, 1991b).

In the genome of salmonid fishes both classes of sequences have already been identified (Moir \& Dixon, 1988). Kido et al. (1991) have characterized three families of SINE sequences derived from tRNA: SmaI, FokI and HpaI families. The salmon SmaI family is restricted to the genomes of chum salmon

*Correspondence. E-mail: egv@sauron.quimica.uniovi.es
(Oncorhynchus keta) and pink salmon (O. gorbuscha). The FokI family is only present in species from the genus Salvelinus, and the HpaI family is present in all the species of the salmonid family. The presence of specific elements of the different families has been used to infer phylogenetic relationships among salmonids (Murata et al., 1996) and more recently to obtain DNA fingerprint patterns (Spruell \& Thorgaard, 1996). In this work, we present the results of our fluorescent in situ hybridization (FISH) experiments on the chromosomes of Atlantic salmon (Salmo salar) and rainbow trout (Oncorhynchus mykiss) using highly repetitive sequences belonging to the $\mathrm{HpaI}$ family.

\section{Materials and methods}

\section{PCR amplifications}

A set of primers based on the Hpa-345 locus of Kokanee salmon (Oncorhynchus nerka adonis) (Murata et al., 1993) was designed for specific amplification of the HpaI family in the genome of Atlantic salmon and rainbow trout. PCR was performed with a Perkin Elmer 2400; the amplification mixture for PCR comprised 5-20 ng of genomic template DNA, $150 \mathrm{pmol}$ of each primer, $200 \mu \mathrm{M}$ of dNTPs and 1 unit of Taq polymerase (Boehringer). Amplification reactions were performed in a thermal cycler (Perkin Elmer 2400) with 30 cycles consisting of $95^{\circ} \mathrm{C}$ for $1 \mathrm{~min}, 58^{\circ} \mathrm{C}$ or $60^{\circ} \mathrm{C}$ for $1 \mathrm{~min}$, and $72^{\circ} \mathrm{C}$ for $1 \mathrm{~min}$. 


\section{Cloning and nucleotide sequence}

The PCR amplified products were treated with kinase, electrophoresed, excised from the gel using a silica matrix (Bio 101) and ligated in the SmaI site of the pUC18 cloning vector, then sequenced in both strands by using the Sequenase Kit (USB) with universal primers. The reaction products were fractionated by electrophoresis on a $6 \%$ polyacrylamide gel. DNA sequences were searched for homologies using the nonredundant amino acid sequence database at the NCBI, with the BLAST program.

\section{Cosmid library}

A total genomic library of Atlantic salmon was constructed in a cosmid vector (sCos-1, Stratagene). Cosmids were isolated by plating out the library at low density and selecting after hybridization with the $H p a$ I unit sequence obtained from the rainbow trout PCR amplification. The probe was labelled by a random priming technique using $\left(\alpha^{32} \mathrm{P}\right) \mathrm{dATP}$ (Amersham). Filters were hybridized at $65^{\circ} \mathrm{C}$ in $3 \times \mathrm{SSPE}$, $0.5 \%$ dried milk, $1 \%$ SDS and $6 \%$ polyethylene glycol overnight, and washed in $1 \times \mathrm{SSC}, 0.1 \% \mathrm{SDS}$ at $65^{\circ} \mathrm{C}$. Positive bands isolated from the genomic cosmid were excised, cloned and sequenced in both strands.

\section{Chromosome preparation and FISH}

Blood was collected from the dorsal artery of 10 large fish species, anaesthetized with MS-222, using a heparinized syringe. Metaphases were obtained from blood cultures as described by Sanchez et al. (1990).
Probes were labelled with biotin-11-dUTP using a nick translation kit (Boehringer). Details of probe preparation, in situ hybridization methods and the analysis of metaphases are described in Pendas et al. (1993a,b).

\section{Results and discussion}

PCR products showed a band of $395 \mathrm{bp}$ in the case of rainbow trout and a band of $255 \mathrm{bp}$ in Atlantic salmon. Cloning and sequencing of four PCR products of each repeat type indicated that rainbow trout contained one unit of the HpaI family, whereas this family was not present in the PCR band resulting from the Atlantic salmon genome amplification. The complete sequences of the two bands are identical to those obtained by Murata et al. (1993) after the amplification of Kokanee salmon and brown trout, respectively (data not shown).

With the aim of locating the $\mathrm{HpaI}$ family in the genome of Atlantic salmon, a total cosmid genomic library from this species was screened with the HpaI unit sequence obtained from the rainbow trout amplification. Positive clones were selected and digested with different restriction enzymes and blotted onto a membrane filter. The filter was then hybridized with the $H p a \mathrm{I}$ unit. Two positive bands resulting after the digestion of one of the positive cosmids with AvaII were cloned and sequenced. The larger fragment cloned was 342 bp long (Fig. 1a) and showed $68 \%$ homology to the coho salmon (Oncorhynchus kisutch) short interspersed element Hpa-51 that is also present in the genome of other Pacific salmon species (Murata et al., 1993). The smaller band, which was 246 bp long (Fig. 1b), showed $82 \%$ identity with the Salmo salar transposon like Tc1-encoded transposase pseudogene isolated by Goodier \& Davidson (1994).

\section{(a)}

seq $342 \mathrm{bp}$

ACCCACCAGTCAAAAGGGGCACACCTATTCATTCAAGGGTTTTTATTTATTTTTTACAATTTTCTACATTGTAG AATAATAGTGAAGACAAGAAAACTATGAAATAACACATATGGAATCATCATGTAGTAACCAAAAAAGATCA ACCAAATCATAATATATTTCATATTTGAGATTCTTCAAAGTAGCCACCCTTTGCCTTGATGAGTTTTGCACAC ACTTGGCATCTCTCAACCAGCTTCATGAGGTAGTCACCTGGATGCATTTCAARGAACAGGTCTCCGTTGTTAA AAGTTAATTTGTGGAATTTCTTCCTCTAGCGTGCACTAGTTGTGTGACAGG

(b)

seq $246 \mathrm{bp}$

CCCACTTTGTTGAAGCCCCTTTGGCAGTGATTACAGCCTGAAGTCTTCTTGGGATGACGCTATAAGTTGGCAC ACCTGTATTTGGGGAGTTTCTCCCATTCTTCTCTGCAGATCCTCTCAAGGCTGTCAGGTTGGACTGAGTGTCG CTGCACAGCTATTTTCAGGTCTCCAGAGGATATTCGCTGGTCAAGTCTGGGCTGCTGGGCCCACTCAAGGACA TTGAGACTTATTCCGAAGCCACTCTGG

Fig. 1 Nucleotide sequence of the two bands cloned from the Atlantic salmon cosmid containing a unit of the HpaI family. Similarities with coho salmon short interspersed element Hpa-51 (a) and Salmo salar transposon (b) are underlined.

(c) The Genetical Society of Great Britain, Heredity, 83, 575-579. 
(a)

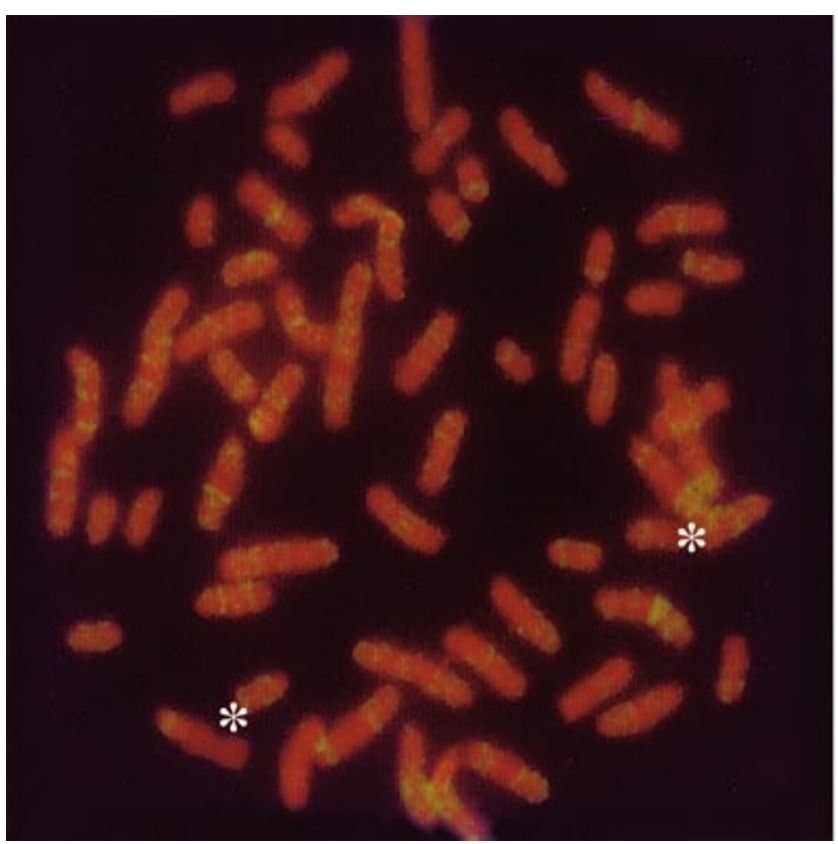

(b)

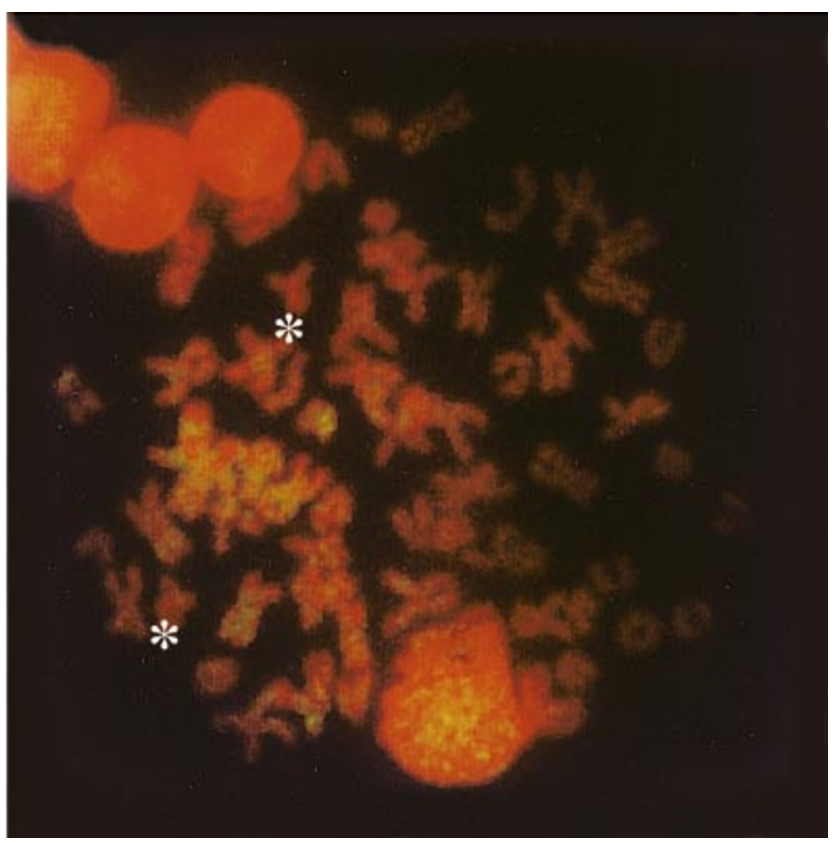

Fig. 2 Fluorescent in situ hybridization (FISH) showing (a) Atlantic salmon chromosomes hybridized with a PCR amplified HpaI SINE fragment from rainbow trout. (b) Rainbow trout chromosomes hybridized with the same probe. Asterisks indicate the rDNAbearing chromosome arms where no hybridization sites were detected.

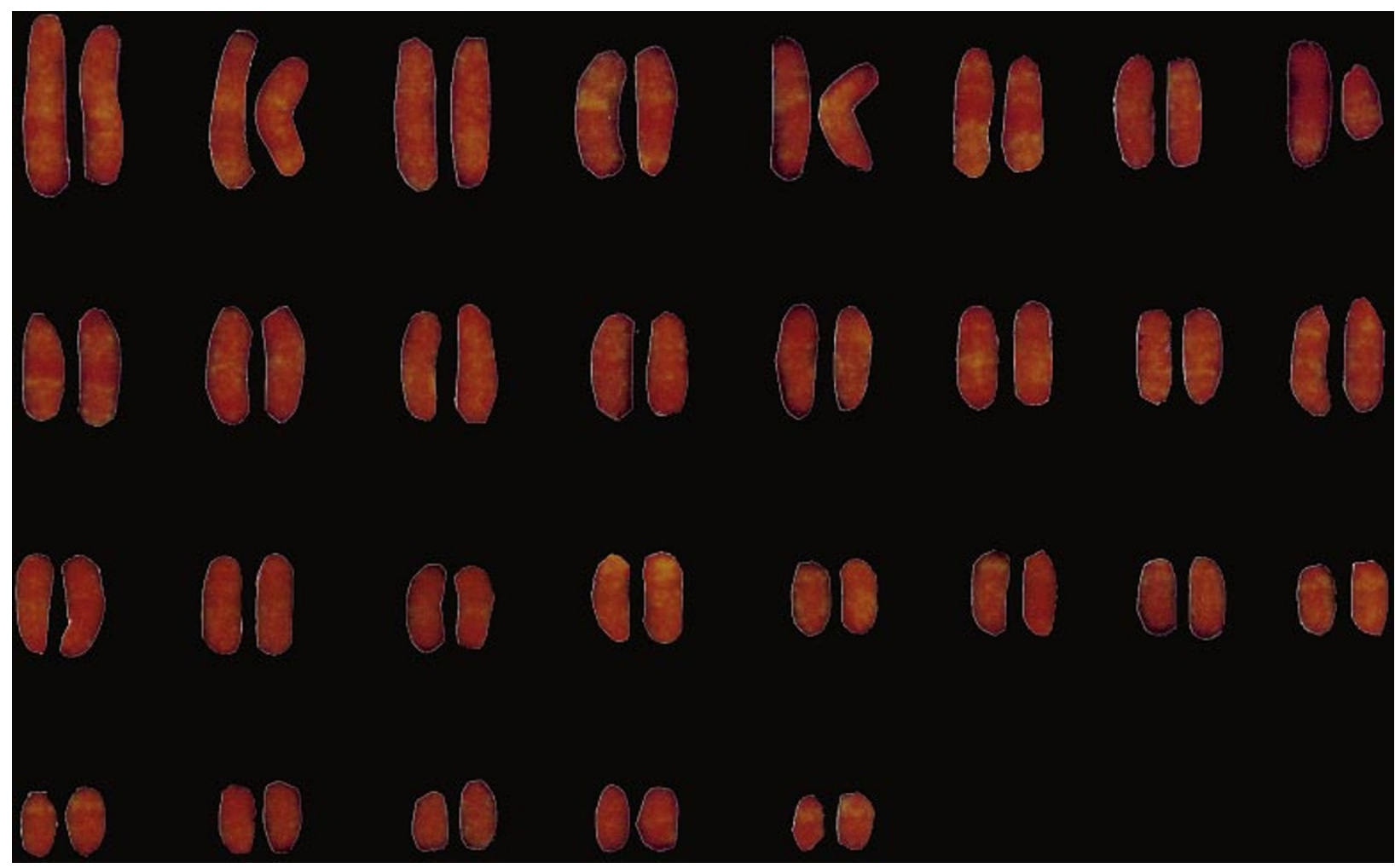

Fig. 3 Homologous pairs identified from the banding pattern of chromosomes shown in Fig. 2(a). This Atlantic salmon karyotype is $2 n=58$.

(C) The Genetical Society of Great Britain, Heredity, 83, 575-579. 
In order to determine the chromosomal distribution of the HpaI family, FISH analysis was carried out in the two species using as probes the PCR-amplified $395 \mathrm{bp}$ fragment from rainbow trout and the $255 \mathrm{bp}$ fragment from Atlantic salmon, the positive cosmid isolated for the $H p a \mathrm{I}$ family and the cloned band from this cosmid that contained a part of the Hpa-51 locus. As expected, hybridization of the PCR product from the Atlantic salmon lacking $H p a$ I family members did not generate any signal in either species. The hybridization signals obtained with the other three probes revealed that the HpaI sequence was interspersed throughout all the chromosome pairs except in the heterochromatin positive areas, for both Atlantic salmon and rainbow trout (see Fig. 2), providing hybridization patterns complementary to the C-banding. Hybridization patterns obtained using the sequence similar to the Hpa-51 locus were identical to the patterns obtained using the whole genomic cosmid. The absence of the SINE sequence in the heterochromatic chromosome arm where the rDNA is located (Pendas et al., 1993a) indicates that the members of this SINE family are located only in the euchromatic regions of the chromosomes.

Nonrandom distribution of SINE elements has been described in human chromosomes. For example, the Alu and $L 1$ families are preferentially found in $\mathrm{R}$ and $\mathrm{G}$ bands, respectively (Korenberg \& Rykowski, 1988); therefore, the distribution of the SINE element found in our work is not surprising. In addition to this particular distribution, we have observed that the $\mathrm{HpaI}$ element contained in the cosmid is located adjacent to a Tc-1 transposon-like element (Goodier \& Davidson, 1994). Spruell \& Thorgaard (1996) have suggested that these two features of SINEs (nonrandom distribution and integration close to other repetitive elements), together with the occurrence of some SINE elements as tandemly repeated arrays (Nagahashi et al., 1991), could be responsible for the production of fingerprinting patterns instead of a smear when these salmon SINE families are used for Southern blot analyses.

The physical distribution of a member of the $\mathrm{HpaI}$ family obtained in this work on Atlantic salmon and rainbow trout chromosomes can probably be extended to the other two SINE families (FokI and SmaI). If assayed on chromosomes from all the other members of the Salmonidae family, FISH of SINE elements could provide new information useful in evolutionary studies (Murata et al., 1993). This method could also contribute to standardizing the Atlantic salmon karyotype, because the fluorescent in situ hybridization of these sequences gives a banding pattern that allows the identification of at least 15 chromosome pairs (Fig. 3).

\section{Acknowledgements}

This work was supported by the Spanish Grant DGICYT PB94-1327.

\section{References}

GOODIER, J. L. AND DAVIDSON, w. S. 1994. Tc1 transposon-like sequences are widely distributed in salmonids. J. Mol. Biol., 241, 26-34.

KIDO, Y., AONO, M., YAMAKI, T., MATSUMOTO, K., MURARA, S. AND OKADA, N. 1991. Shaping and reshaping of salmonid genomes by amplification of tRNA-derived retroposons during evolution. Proc. Natl. Acad. Sci. U.S.A., 88, 2326-2330.

KORENBERG, J. R. AND RYKOWSKI, M. C. 1988. Human genome organization: Alu, LINEs and the molecular structure of metaphase chromosome bands. Cell, 53, 391-400.

MOIR, R. D. AND DIXON, G. H. 1988. A repetitive DNA sequence in the salmonid fishes similar to a retroviral long treminal repeat. J. Mol. Evol., 27, 1-7.

MURATA, S., TAKASAKI, N., SAITOH, M. AND OKADA, N. 1993. Determination of the phylogenetic relationships among Pacific salmonids by using short interspersed elements (SINEs) as temporal landmarks of evolution. Proc. Natl. Acad. Sci. U.S.A., 90, 6995-6999.

MURATA, S., TAKASAKI, N., SAITOH, M., TACHIDA, H. AND OKADA, N. 1996. Details of retropositional genome dynamics that provide a rationale for a generic division: the distinct branching of all the Pacific salmon and trout (Oncorhynchus) from the Atlantic salmon and trout (Salmo). Genetics, 142, 915-926.

NAGAHASHI, S., ENDOH, H., SUZUKI, Y. AND OKADA, N. 1991. Characterization of a tandemly repeated DNA sequence family originally derived by retroposition of tRNA glu in the newt. J. Mol. Biol., 222, 391-404.

OHSHIMA, K. R., KOISHI, M., MATSUO, M. AND OKADA, N. 1993. Several short interspersed repetitive elements (SINEs) in distant species may have originated from a common ancestral retrovirus: characterization of a squid SINE and a possible mechanism for generation of tRNA-derived retroposons. Proc. Natl. Acad. Sci. U.S.A., 90, 6260-6264.

OKADA, N. 1991a. SINEs: Short interspersed repeated elements of the eukaryotic genome. Trends Ecol. Evol., 6, 358-361.

okADA, N. 1991b. SINEs. Curr. Opin. Genet. Dev., 1, 498-504. OKADA, N. AND OHSHIMA, K. 1995. Evolution of tRNA-derived SINEs. In: Maraia, R. J. (ed.) The Impact of Short Interspersed Elements (SINEs) on the Host Genome, pp. 61-79. R. G. Landes, Austin, TX.

PENDAS, A. M., MORAN, P. AND GARCIA-VAZQUEZ, E. 1993 a. Ribosomal RNA genes are interspersed throughout a heterochromatic chromosome arm in Atlantic salmon. Cytogenet. Cell Genet., 63, 128-130.

PENDAS, A. M., MORAN, P. AND GARCIA-VAZQUEZ, E. 1993b. Multi-chromosomal location of ribosomal RNA genes and heterochromatic association in brown trout. Chromosome Res., 1, 63-67. 
SANCHEZ, L., MARTINEZ, P., VIÑAS, A. AND BOUZA, C. 1990. Analysis of the structure and variability of nucleolar organizer regions of Salmo trutta by C-, Ag-, and restriction endonuclease banding. Cytogenet. Cell Genet., 54, 6-9.

SINGER, M. F. 1982. SINEs and LINEs: Highly repeated short and long interspersed sequences in mammalian genomes. Cell, 28, 433-434.
SMIT, A. F. 1996. The origin of interspersed repeats in the human genome. Curr. Opin. Genet. Dev., 6, 743-748.

SPRUELL, P. AND THORGAARD, G. H. 1996. SINE sequences detect DNA fingerprints in salmonid fishes. Heredity, 76, 317-324.

ULLU, E. AND TSCHUDI, C. 1984. Alu sequences are processed 7SL RNA genes. Nature, 312, 171-172. 